Z. Phys. Chem. 217 (2003) 707-722

(C) by Oldenbourg Wissenschaftsverlag, München

\title{
Temperature Dependence of Viscosity and Density of cis-1,4/trans-1,3-Dimethylcyclo- hexane and Several other Commonly Used Organic Solvents
}

\author{
By A. A. Ruth ${ }^{1, *}$, H. Lesche ${ }^{2}$, and (the late) B. Nickel ${ }^{2}$ \\ ${ }^{1}$ Department of Chemistry, National University of Ireland, University College Cork, \\ Cork, Ireland \\ 2 Max-Planck Institut für Biophysikalische Chemie, Abteilung Spektroskopie und \\ Photochemische Kinetik, Am Fassberg 11, D-37077 Göttingen, Germany
}

(Received December 18, 2002; accepted January 16, 2003)

Dynamic Viscosity / Density / Temperature Dependence /

Glass Transition / cis-1,4/trans-1,3-Dimethylcyclohexane / Isooctane / 2,2,4-Trimethylpentane / Cyclopentane / Isopentane / 2-Methylpentane / Isohexane / 1-Propanol / n-Propyl Alcohol / Alkanes

The dynamic viscosity $(\eta)$ of the glass-forming 50:50 mixture of cis-1,4/trans-1,3dimethylcyclohexane (ct-DMCH) was measured from $293 \mathrm{~K}$ down to $\approx 126 \mathrm{~K}$ where $\eta \sim 1.2 \times 10^{6} \mathrm{mPas}$. The viscosity measurements of several other commonly used solvents cover the range from $293 \mathrm{~K}$ down to $\approx 148 \mathrm{~K}\left(\eta \sim 1.4 \times 10^{4} \mathrm{mPas}\right)$ for 1-propanol (1-Prop), to $\approx 118 \mathrm{~K}\left(\eta \sim 2.5 \times 10^{2} \mathrm{mPas}\right)$ for 2-methylpentane (2-MP), to $\approx 167 \mathrm{~K}(\eta \sim 10.0 \mathrm{mPas})$ for isooctane (Isooct), to $\approx 183 \mathrm{~K}(\eta \sim 2.8 \mathrm{mPas})$ for cyclopentane $(\mathrm{CP})$ and down to $\approx 98 \mathrm{~K}\left(\eta \sim 4.6 \times 10^{2} \mathrm{mPas}\right)$ for the $30: 70$ mixture of cyclopentane/isopentane $(\mathrm{CP} / \mathrm{IP})$. The density $(\varrho)$ of all solvents was measured correspondingly over appropriate temperature ranges.

For the solvents studied here, the temperature dependence of the viscosity can be represented by a single Arrhenius term down to $\sim 180 \mathrm{~K}$. Over a wider temperature range down to $\sim 118 \mathrm{~K}$ the sum of two Arrhenius terms is required, and in the low temperature regime a Vogel-Tammann-Fulcher expression is necessary to adequately describe the temperature dependence of the dynamic viscosity.

\section{Introduction}

A vast variety of chemical and physical processes in solution are based on transport processes and are therefore strongly dependent on the viscosity of

* Corresponding author. E-mail: a.ruth@ucc.ie 
the solvent. The temperature dependence of the viscosity is hence regarded as one of the fundamental properties of a liquid which determines the timedependence of relaxation functions as well as the size of transport coefficients. Therefore accurate viscosity data of commonly used solvents as a function of temperature are of general interest in many research areas dealing with relaxation and reaction dynamics in molecular liquids.

In this publication the temperature dependence of the dynamic viscosity, $\eta$, and density, $\varrho$, of four frequently used solvents and two solvent mixtures is presented (all liquids are outlined in detail in the experimental section). The viscosity values for the one-component liquids either supplement the data in the literature or they are compared with older measurements where available for the respective liquid. For the solvent mixtures no temperature-dependent viscosity measurements exist.

The viscosity of the mixture cis-1,4/trans-1,3-dimethylcyclohexane (ct-DMCH) is particularly important to us, since it is used in experiments to study the kinetics of the $p$-type delayed fluorescence of anthracene in viscous solution [1]. These measurements are to demonstrate the validity of the application of an adequate form of Smoluchowski's theory to diffusion controlled triplet-triplet annihilation (TTA), which is strongly dependent on the viscosity of the solvent at low temperatures. $\mathrm{Ct}-\mathrm{DMCH}$ was chosen as solvent for the delayed fluorescence measurements for the following reasons: (a) It is a glassforming liquid; (b) It is photochemically stable; (c) The solubility of polycyclic aromatic hydrocarbons (like anthracene) is high in ct-DMCH even at high viscosity; (d) The formation of microcrystals is strongly suppressed in ct-DMCH at low temperature - this essential feature is important since microcrystals can severely distort the spectral dependence of the delayed fluorescence. By applying Smoluchowki's theory to TTA in viscous solution, the diffusion coefficient $D_{\mathrm{T}}$ of triplet anthracene can be extracted from the time-dependence of the delayed fluorescence [2]. The dependence of $D_{\mathrm{T}}$ on $T / \eta$ according to the Stokes-Einstein relation serves as a crucial test for the validity of Smoluchowki's formula. This motivated the measurement of $\eta$ for ct-DMCH with the highest possible accuracy over $\approx 6$ orders of magnitude $(T=293 \ldots 126 \mathrm{~K})$. The dependence of the viscosity on the composition of the mixture is also briefly discussed.

\section{Experiment}

\subsection{Solvent preparation}

The solvents and solvent mixtures reported in this publication are: 1-propanol (1-Prop, Aldrich 99.7\%, freezing point (f.p.) $147.0 \mathrm{~K}$ [3]), isooctane ${ }^{1}$ (Isooct,

\footnotetext{
${ }^{1}$ Isooctane is also known as 2,2,4-trimethylpentane; 2-methylpentane is also known as isohexane.
} 
Aldrich HPLC 99.9\%. f.p. 165.8 K [3]), cyclopentane (CP, Merck Uvasol, f.p. $179.3 \mathrm{~K}$ [3]), 2-methylpentane ${ }^{1}$ (2-MP, Aldrich $99+\%$, f.p. $119.5 \mathrm{~K}$ [3]), 30:70 mixture by weight of cyclopentane:isopentane $(\mathrm{CP} / \mathrm{IP}$, isopentane from Merck Uvasol, \#6056) and the 50:50 mixture by volume of cis-1,4 and trans-1,3-dimethyIcyclohexane (ct-DMCH, Aldrich 99\%, see diagram). All solvents except for ct-DMCH were fractionally destilled over a $1 \mathrm{~m}$ column (feedback ratio 1:100), chromatographically purified and dried with basic aluminium oxide and degassed by a sufficient number of freeze-pump-thaw cycles.

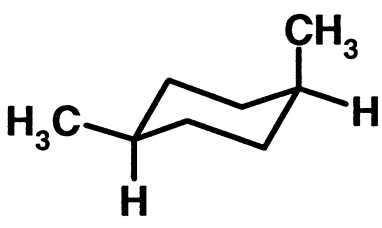

cis-1,4-dimethylcyclohexane

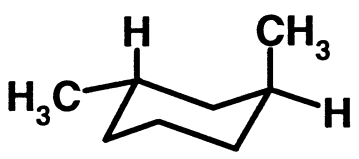

trans-1,3-dimethylcyclohexane

\subsection{Viscosimetry}

The viscosity was measured by capillary viscosimetry [4-6] using KPG Ubbelohde viscosimeters (types $0,0 \mathrm{c}, \mathrm{I}, \mathrm{Ic}, \mathrm{He}, \mathrm{IIIc}, \mathrm{IVc}$, and V). The viscosimeters are calibrated by the manufacturer with an accuracy of $\pm 0.1 \%$. Details on the evaluation procedure of viscosity values including adequate correction procedures and a description of several modifications of the standard capillary method regarding the filling procedure and thermalization of the sample, are described in previous publications [7, 8]. All general estimates concerning the size of systematic error limits of the apparati are given in these publications and apply in the present work (except for the estimated temperature accuracy, which is described in the following paragraph).

For the viscosity and density measurements with 2-MP, CP, and CP/IP a home-built prototype cryostat (A) was used, which is described in detail in [7]. The temperature control consisted of a calibrated Pt-100 resistance thermometer (Lauda R46) in combination with a control unit (Haake TP24) (also $c f$. [8]). The spatial homogeneity of the temperature in this cryostat is due to a temperature gradient ${ }^{2}$ that accounts for a temperature accuracy of approximately $\pm 0.15 \mathrm{~K}$. This accuracy ultimately determines the accuracy of the viscosities obtained for $2-\mathrm{MP}, \mathrm{CP}$, and $\mathrm{CP} / \mathrm{IP}$.

For the viscosity and density measurements of ct-DMCH, 1-Prop, and Isooct, however, cryostat (A) was replaced by a different home-built cryostat (B), with improved temperature stability. This cryostat is described in [8]. As

${ }^{2}$ The temperature at the bottom of the copper block inside the cryostat is colder than the top by $\approx 0.3 \mathrm{~K}$. 
opposed to the control unit outlined in [8] (the same as mentioned above) a digital temperature controller (Lakeshore DRC 93CA) was used in the present work. The temperature stability in this cryostat is estimated to be of the same order of accuracy as the digital measurement of the absolute temperature of $\pm 0.05 \mathrm{~K}$, which ultimately determines the accuracy of the viscosities obtained for ct-DMCH, 1-Prop and Isooct.

The density of all liquids was measured using standard pycnometers. The respective procedure is described in detail in Section 2.5 of [8].

\section{Results}

Figs. 1-3 show the experimental dynamic viscosity data of all liquids as a function of $T$. Fig. 1 shows $\eta(T)$ of the mixture ct-DMCH for three different mole fractions of the cis-1,4 and trans-1,3 components, i.e. the 50:50 (cis:trans) mixture over the temperature range $293 \ldots 126 \mathrm{~K}$, and the $45: 55$ as well as the 55:45 mixtures (in the high-viscosity range only, $143 \ldots 126 \mathrm{~K}$ ). The mixtures 50:50, 45:55, and 55:45 are refered to as ct-DMCH(1), (2), and (3), respectively (Table 1). Fig. 2 shows $\eta(T)$ of the 30:70 mixture CP/IP from 293 to $98 \mathrm{~K}$, as well as of the $27: 73$ mixture of $\mathrm{CP} / \mathrm{IP}$ (in the temperature range $113 \ldots 99 \mathrm{~K}$ only). These mixtures are refered to as $\mathrm{CP} / \mathrm{IP}(1)$ and (2), respectively (Table 1). Fig. 3 shows $\eta(T)$ of 1-Prop, Isooct, 2-MP and CP.

We retained the empirical description of the data $\eta(T)$ [mPas] given in [8],

$$
\begin{aligned}
& \eta(T) \approx f(T)=f_{1}(T)+f_{2}(T)+f_{3}(T)=A_{1} \exp \left(E_{1} / R T\right) \\
& +A_{2} \exp \left(E_{2} / R T\right)+A_{3} \exp \left[E_{3} / R\left(T-T_{0}\right)\right],
\end{aligned}
$$

since it proved satisfactory for all solvents and temperature ranges studied. Like any alternative analytical expression for the temperature dependence of the viscosity $\eta$ of organic liquids [3,9-11], the validity and accuracy of Eq. (1) depends on the viscosity range $\left(\eta_{\min }, \eta_{\max }\right)$ that is to be covered. When $\eta_{\min }$ is small $(<1 \mathrm{mPas})$ and $\eta_{\max } / \eta_{\min }$ is $<10$ a single Arrhenius term $f_{1}(T)$ is often sufficient to describe $\eta(T)$ with an accuracy of $\approx 1 \%$ [3]. In the high-viscosity range of glass-forming supercooled liquids a single VogelTammann-Fulcher term $f_{3}(T)$ can be sufficient to describe the temperature dependence $[9,12]$ (see rows 2,3 and 7 in Table 1). In the intermediate viscosity range $\left(10<\eta_{\max } / \eta_{\min }<10^{4}\right.$, assuming a small $\left.\eta_{\min }\right)$ either two Arrhenius terms, $f_{1}+f_{2}$, or the full Eq. (1) are required to represent $\eta(T)$ with sufficient accuracy, depending on the liquid.

In the case of $\mathrm{CP}$ only one Arrhenius term $f_{1}$ was required to decribe $\eta(T)$, two Arrhenius terms $f_{1}$ and $f_{2}$ were used for 1-Prop and Isooct, and for 2-MP, CP/IP and ct-DMCH the full Eq. (1) was applied to represent the dependence of $\eta$ on temperature over the entire range studied. The individual 


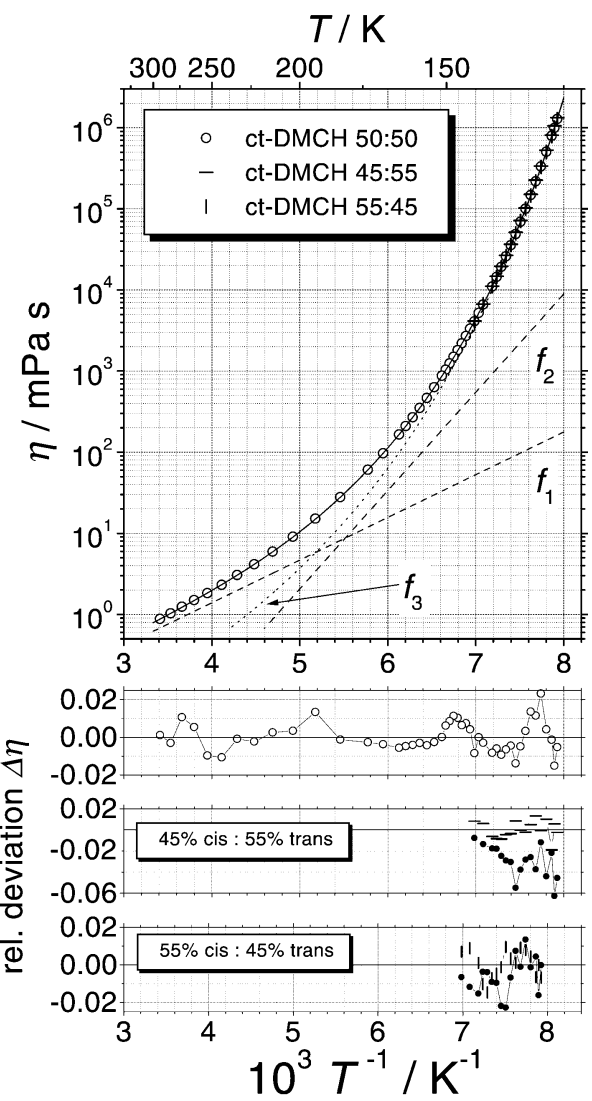

Fig. 1. Arrhenius plot of the viscosity $\eta(T)$ for ct-DMCH(l), measured in cryostat (B) [8], i.e. $\Delta T \sim \pm 0.05 \mathrm{~K}$. The solid lines $f(T)$ are calculated with Eq. (1) and the parameter values in Table 1 . The two dashed lines, $f_{1}, f_{2}$, and the dotted line $f_{3}$ represent the three summands of $f(T)$ in Eq. (1) for ct-DMCH(1). The quantity $\Delta \eta$ in the lower part of the figure (symbols "o", "-" and "" for ct-DMCH(1) ... (3)) is the relative deviation of $f(T)$ from $\eta(T): \Delta \eta=[\eta(T)-f(T)] / \eta(T)$. Symbol " $\bullet$ " is used for the relative deviations $\Delta \eta=\left(\eta_{\mathrm{DMCH}(1)}-\eta_{\mathrm{ct}-\mathrm{DMCH}(k)}\right) / \eta_{\mathrm{ct}-\mathrm{DMCH}(1)}(k=2,3)$.

contributions of the Arrhenius terms $f_{1}(T)$ and $f_{2}(T)$ as well as the VogelTammann-Fulcher (VTF) term $f_{3}(T)$ are explicitly shown for ct-DMCH in Fig. 1 and CP/IP in Fig. 2. All parameters used for the best fit of Eq. (1) to the experimental data are given in Table 1, which also includes the respective minimum temperatures, $T_{\min }$, of the viscosity measurements. Calculated values of $\log _{10}(f(T) / \mathrm{mPa}$ s) are listed in Table 2 and measured minimum and maximum viscosities, $\eta_{\min }$ and $\eta_{\max }$, as well as the ratios $\eta_{\max } / \eta_{\min }$ are stated in the caption of Table 2 for all liquids. The lower parts of Figs. 1-3 show the relative deviation $\Delta \eta=[\eta(T)-f(T)] / \eta](T)$ of the fitted curve $f(T)$ from the values $\eta(T)$, 


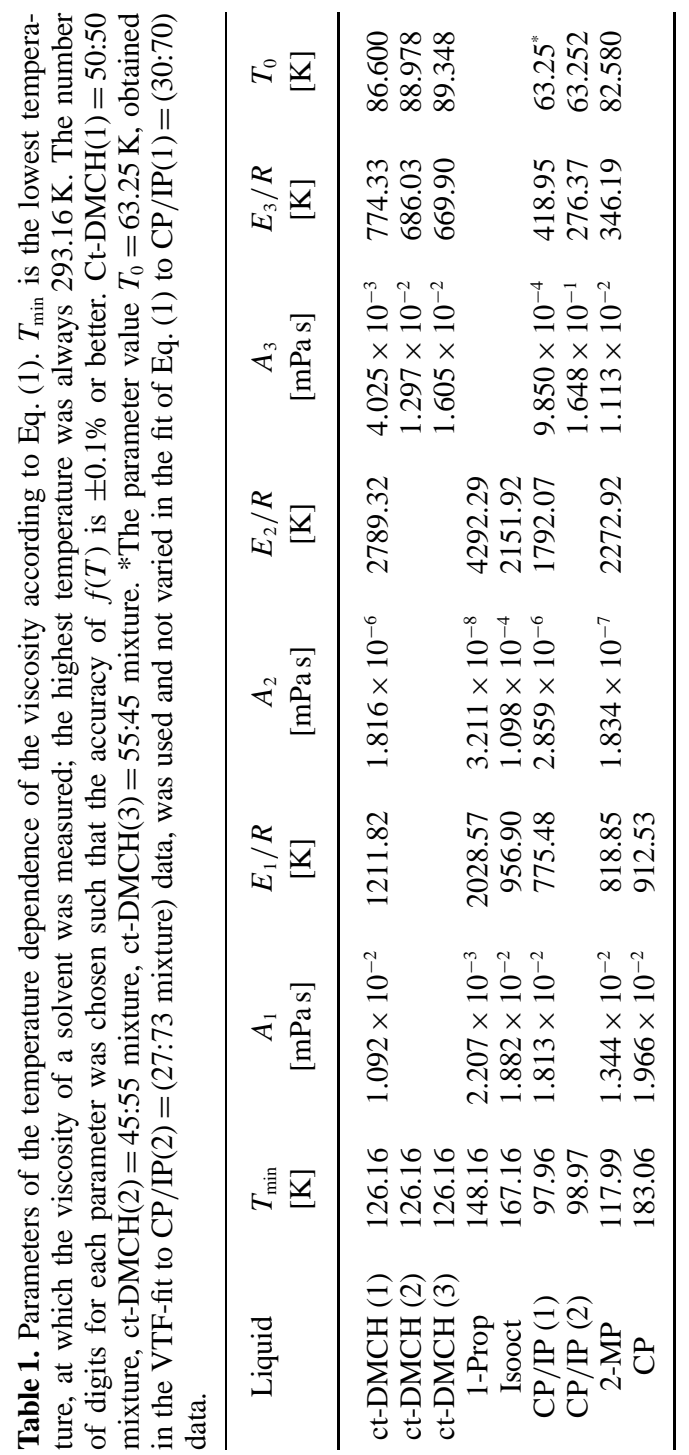

which is for the majority of data-points smaller than $1 \%$, in all cases smaller than $2 \%$.

The apparently linear temperature dependence of the experimental density data $\varrho(T)$ is shown in Fig. 4. Based on Refs. [7,8] we used a third-order polynomial for the empirical description of $\varrho(T)$ :

$$
\varrho(T) \approx g(T)=B_{0}+B_{1} T+B_{2} T^{2}+B_{3} T^{3} .
$$




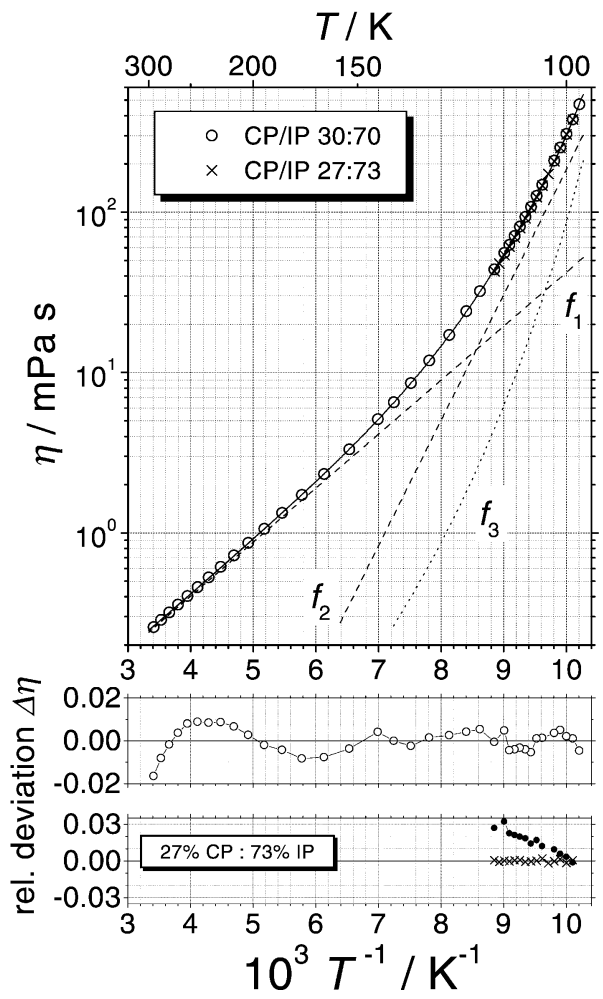

Fig. 2. Arrhenius plot of the viscosity $\eta(T)$ for CP/IP(1), measured in cryostat (A) [7], i.e. $\Delta T \sim \pm 0.15 \mathrm{~K}$. The solid lines $f(T)$ are calculated with Eq. (1) and the parameter values in Table 1 . The two dashed lines, $f_{1}, f_{2}$, and the dotted line $f_{3}$ represent the three summands of $f(T)$ in Eq. (1) for $\mathrm{CP} / \mathrm{IP}(1)$. The quantity $\Delta \eta$ in the lower part of the figure (symbols " $\times$ " for $\mathrm{CP} / \mathrm{IP}(2)$ ) is the relative deviation of $f(T)$ from $\eta(T)$ : $\Delta \eta=[\eta(T)-f(T)] / \eta(T)$. Symbol "•" is used for the relative deviations $\Delta \eta=\left(\eta_{\mathrm{CP} / \mathrm{IP}(1)}-\right.$ $\left.\eta_{\mathrm{CP} / \mathrm{IP}(2)}\right) / \eta_{\mathrm{CP} / \mathrm{IP}(1)}$.

As shown in the lower part of the figure, the magnitude of the relative deviation $\Delta \varrho=[\varrho(T)-g(T)] / \varrho(T)$ of $g(T)$ from $\varrho(T)$ is generally smaller than $0.05 \%$ (with only two exceptions for CP/IP). The parameters used for the fit of Eq. (2) to the experimental data are listed in Table 3.

\section{Discussion}

Depending on the cooling conditions, for instance through very rapid cooling, every liquid can in principle be supercooled below its freezing (crystallization) point into a non-equilibrium amorphous, so-called "glassy" state. Since for our measurements the cooling rate was very small, the lowest temperatures 
Table 2. Values of $\operatorname{Lg} \eta \equiv \log _{10}(f(T) / \mathrm{mPas})$ calculated with Eq. (1) using the parameters in Table 1 at temperatures given in column 2. The values in brackets are extrapolated. The minimum and maximum measured values as well as their ratio $\left(\eta_{\min }[\mathrm{mPas}]\right.$, $\left.\eta_{\max }[\mathrm{mPas}], \eta_{\max } / \eta_{\min }\right)$ are given for all liquids studied: 50:50 ct-DMCH $(\approx 0.878$, $\left.\approx 1275350, \approx 1.5 \times 10^{6}\right), \mathrm{ct}-\mathrm{DMCH}(2)(\approx 4126, \approx 1333440, \approx 323)$, ct-DMCH(3) $(\approx 4121, \approx 1275560, \approx 309)$; 1-Prop $(\approx 2.228, \approx 14609, \approx 7000)$; Isooct $(\approx 0.508$, $\approx 10.0, \approx 20) ; \mathrm{CP} / \mathrm{IP}(1)(\approx 0.258, \approx 470, \approx 1900), \mathrm{CP} / \mathrm{IP}(2)(\approx 42.7, \approx 378, \approx 9) ; 2-\mathrm{MP}$ $(\approx 0.275, \approx 253.2, \approx 920) ; \mathrm{CP}(\approx 0.439, \approx 2.87, \approx 7)$.

\begin{tabular}{|c|c|c|c|c|c|c|c|}
\hline $\begin{array}{c}T^{-1} \\
{\left[10^{3} \mathrm{~K}^{-1}\right]}\end{array}$ & $\begin{array}{c}T \\
{[\mathrm{~K}]}\end{array}$ & $\begin{array}{c}\text { ct-DMCH } \\
\operatorname{Lg} \eta\end{array}$ & $\begin{array}{c}\text { 1-Prop } \\
\operatorname{Lg} \eta\end{array}$ & $\begin{array}{l}\text { Isooct } \\
\operatorname{Lg} \eta\end{array}$ & $\begin{array}{c}\mathrm{CP} / \mathrm{IP} \\
\mathrm{Lg} \eta\end{array}$ & $\begin{array}{l}\text { 2-MP } \\
\operatorname{Lg} \eta\end{array}$ & $\begin{array}{c}\mathrm{CP} \\
\operatorname{Lg} \eta\end{array}$ \\
\hline 3.4 & 294.12 & -0.0632 & 0.3406 & -0.2978 & -0.5840 & -0.5605 & -0.3590 \\
\hline 3.6 & 277.78 & 0.0530 & 0.5175 & -0.2109 & -0.5163 & -0.4915 & -0.2797 \\
\hline 3.8 & 263.16 & 0.1727 & 0.6950 & -0.1230 & -0.4485 & -0.4219 & -0.2005 \\
\hline 4.0 & 250.00 & 0.2964 & 0.8731 & -0.0338 & -0.3805 & -0.3515 & -0.1212 \\
\hline 4.2 & 238.10 & 0.4254 & 1.0524 & 0.0569 & -0.3123 & -0.2803 & -0.0419 \\
\hline 4.4 & 227.27 & 0.5608 & 1.2333 & 0.1494 & -0.2439 & -0.2082 & 0.0373 \\
\hline 4.6 & 217.39 & 0.7039 & 1.4169 & 0.2441 & -0.1752 & -0.1351 & 0.1166 \\
\hline 4.8 & 208.33 & 0.8563 & 1.6044 & 0.3416 & -0.1061 & -0.0609 & 0.1958 \\
\hline 5.0 & 200.00 & 1.0197 & 1.7979 & 0.4423 & -0.0367 & 0.0147 & 0.2751 \\
\hline 5.2 & 192.31 & 1.1955 & 1.9999 & 0.5468 & 0.0333 & 0.0919 & 0.3544 \\
\hline 5.4 & 185.19 & 1.3853 & 2.2139 & 0.6556 & 0.1039 & 0.1711 & 0.4336 \\
\hline 5.6 & 178.57 & 1.5906 & 2.4435 & 0.7693 & 0.1752 & 0.2528 & $(0.5129)$ \\
\hline 5.8 & 172.41 & 1.8129 & 2.6923 & 0.8883 & 0.2473 & 0.3374 & $(0.5922)$ \\
\hline 6.0 & 166.67 & 2.0538 & 2.9626 & (1.0132) & 0.3205 & 0.4257 & $(0.6714)$ \\
\hline 6.2 & 161.29 & 2.3157 & 3.2550 & (1.1441) & 0.3950 & 0.5186 & $(0.7507)$ \\
\hline 6.4 & 156.25 & 2.6013 & 3.5676 & (1.2813) & 0.4708 & 0.6171 & $(0.8299)$ \\
\hline 6.6 & 151.52 & 2.9148 & 3.8972 & (1.4245) & 0.5484 & 0.7224 & \\
\hline 6.8 & 147.06 & 3.2610 & $(4.2402)$ & (1.5737) & 0.6279 & 0.8360 & \\
\hline 7.0 & 142.86 & 3.6458 & (4.5929) & & 0.7098 & 0.9595 & \\
\hline 7.2 & 138.89 & 4.0752 & $(4.9525)$ & & 0.7943 & 1.0947 & \\
\hline 7.4 & 135.14 & 4.5553 & & & 0.8818 & 1.2437 & \\
\hline 7.6 & 131.58 & 5.0921 & & & 0.9728 & 1.4087 & \\
\hline 7.8 & 128.21 & 5.6922 & & & 1.0675 & 1.5924 & \\
\hline 8.0 & 125.00 & $(6.3639)$ & & & 1.1665 & 1.7982 & \\
\hline 8.2 & 121.95 & (7.1180) & & & 1.2700 & 2.0300 & \\
\hline 8.4 & 119.05 & (7.9688) & & & 1.3784 & 2.2935 & \\
\hline 8.6 & 116.28 & $(8.9355)$ & & & 1.4922 & (2.5954) & \\
\hline 8.8 & 113.64 & (10.0430) & & & 1.6116 & (2.9440) & \\
\hline 9.0 & 111.11 & & & & 1.7372 & (3.3486) & \\
\hline 9.2 & 108.70 & & & & 1.8693 & (3.8198) & \\
\hline 9.4 & 106.38 & & & & 2.0088 & (4.3697) & \\
\hline 9.6 & 104.17 & & & & 2.1566 & & \\
\hline 9.8 & 102.04 & & & & 2.3140 & & \\
\hline 10.0 & 100.00 & & & & 2.4832 & & \\
\hline 10.2 & 98.04 & & & & 2.6669 & & \\
\hline 10.4 & 96.15 & & & & (2.8684) & & \\
\hline 10.6 & 94.34 & & & & (3.0919) & & \\
\hline 10.8 & 92.59 & & & & (3.3417) & & \\
\hline 11.0 & 90.91 & & & & $(3.6215)$ & & \\
\hline 11.2 & 89.29 & & & & (3.9339) & & \\
\hline
\end{tabular}




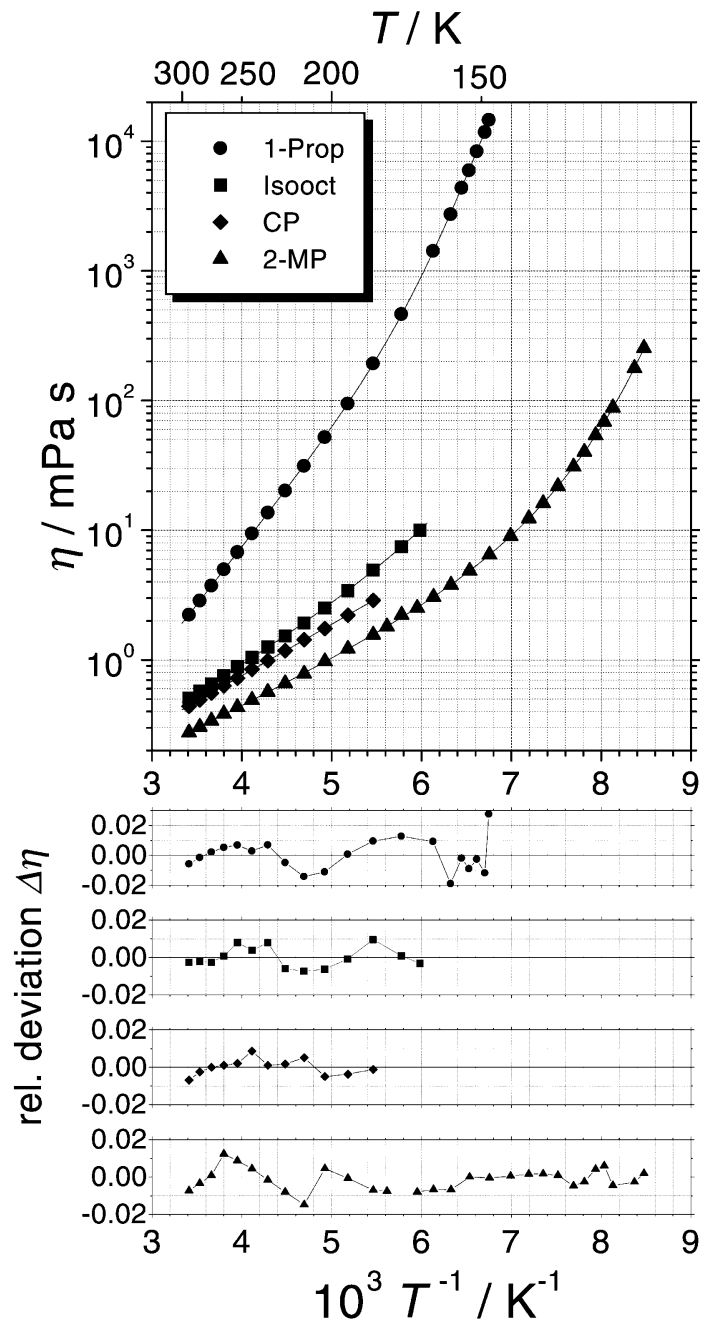

Fig. 3. Arrhenius plot of the viscosity $\eta(T)$ for 1-Prop, Isooct, CP and 2-MP. The solid lines $f(T)$ are calculated with Eq. (1) and the parameter values in Table 1. The quantity $\Delta \eta$ in the lower part of the figure is the relative deviation of $f(T)$ from $\eta(T): \Delta \eta=$ $[\eta(T)-f(T)] / \eta(T)$. 1-Prop and Isooct measured in cryostat (A) [7], i.e. $\Delta T \sim \pm 0.15 \mathrm{~K}$; 2-MP and $\mathrm{CP}$ measured in cryostat (B) [8], i.e. $\Delta T \sim \pm 0.05 \mathrm{~K}$.

at which the viscosity of the single component liquids was determined, was always one degree or more above the known freezing points. The mixtures $\mathrm{CP} / \mathrm{IP}$ and ct-DMCH, however, are known to be glass-forming liquids. Particularly ct$\mathrm{DMCH}$ as a liquid with different conformers may even undergo multiple glass transitions [15] and form, besides a glassy liquid, a so-called plastic crystalline 


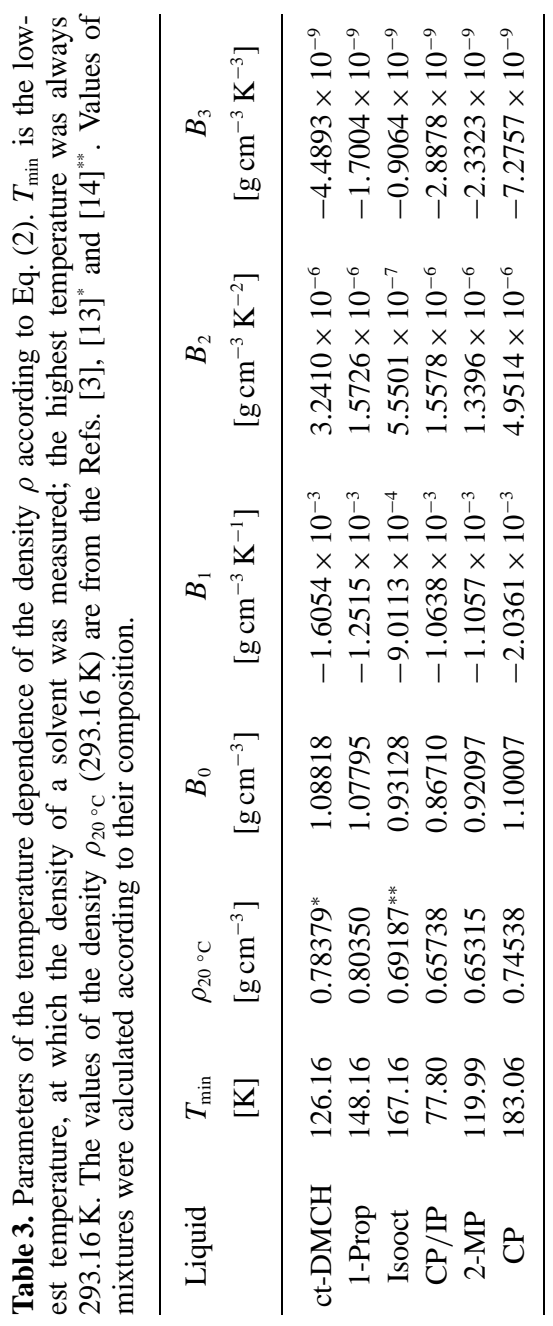

phase in the glass transition [16]. The highest viscosity at the lowest temperature in our measurements with ct-DMCH, $\eta(126 \mathrm{~K}) \approx 1.27 \times 10^{6} \mathrm{mPas}$, is however many orders of magnitude smaller than $\eta$ at the glass transition temperature, $T_{\mathrm{g}} .{ }^{3}$ The deviation from a thermally activated (Arrhenius-) behaviour of $\eta$ somewhat above $126 \mathrm{~K}$, which is demonstrated by the fact that a VTF-term is required to adequately fit the temperature dependence, shows that the onset of the glass transition is already in this temperature region. The

${ }^{3} T_{\mathrm{g}}$ is generally defined as the temperature, where the viscosity reaches a value of $\eta\left(T_{\mathrm{g}}\right)=10^{15} \mathrm{mPa}$. Viscosities at $T_{\mathrm{g}}$ imply relaxation times in excess of $100 \mathrm{~s}$. 


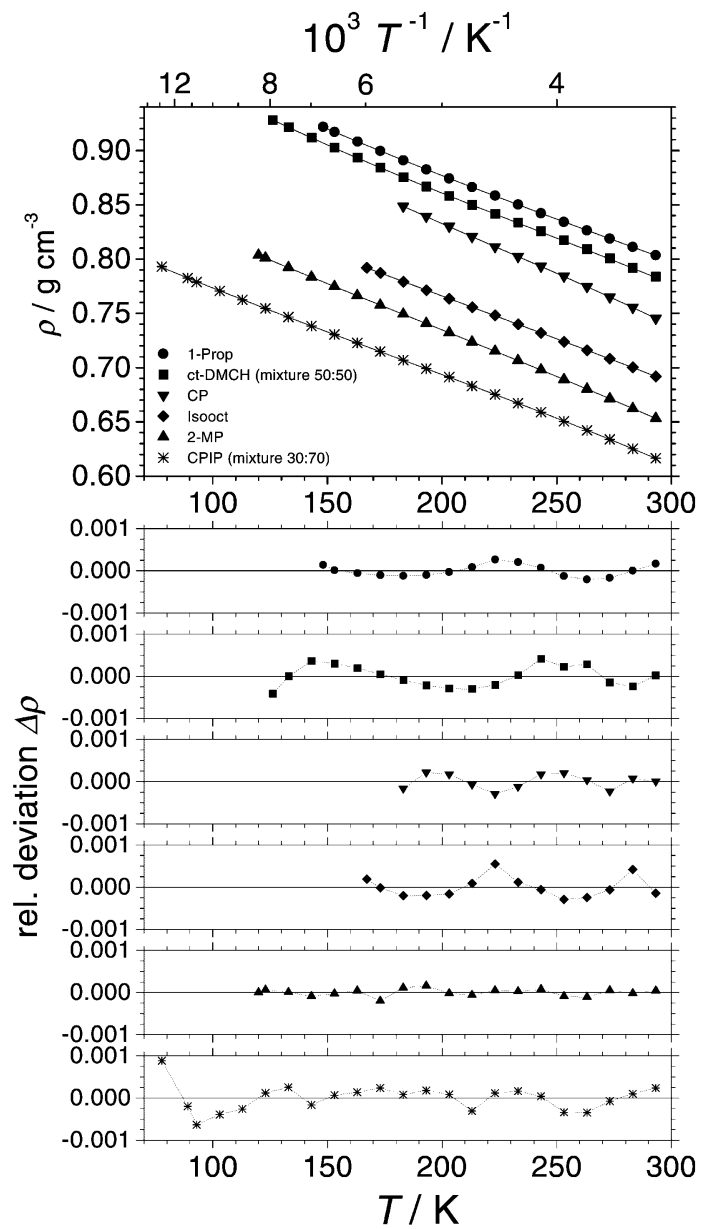

Fig. 4. Linear plot of the density $\rho(T)$. The solid lines $g(T)$ are calculated with Eq. (2) and the parameter values in Table 3 . The quantity $\Delta \rho$ in the lower part of the figure is the relative deviation of $g(T)$ from $\rho(T): \Delta \rho=[\rho(T)-g(T)] / \rho(T)$.

parameter $T_{0}$ in the VTF term in Eq. (1) is typically found to be $\sim 20 \ldots 30 \mathrm{~K}$ below the respective glass transition temperature $T_{\mathrm{g}}$. At temperatures below $T_{0}$ diffusion based processes and molecular reorientation are completely frozen. A very rough estimate of $T_{\mathrm{g}}$ by extrapolating $\eta$ to $10^{15} \mathrm{mPa}$, yields $T_{\mathrm{g}} \sim 106 \mathrm{~K}$ for ct-DMCH, which is in accord with the value of $T_{0} \approx 86 \mathrm{~K}$ in Table 1. In comparison, a temperature for the plastic crystalline phase transition $T_{\mathrm{g}^{\prime}}$ of the solvent cis-1,2-dimethylcyclohexane was found to be $107.8 \mathrm{~K}$ [15], which is surprisingly close to the very approximate value of $T_{\mathrm{g}}$ of ct-DMCH estimated here. The value of $T_{0} \approx 73 \mathrm{~K}$ for cis-1,2-dimethylcyclohexane, 
based on dielectric relaxation measurements in [16] does not compare as well.

The ratio of $E_{3} /\left(R T_{0}\right) \equiv F$ (in the following referred to as "fragility parameter") can be used to classify liquids in terms of strong and fragile glass formers according to Angell $[17,18]$. For the liquids ct-DMCH $(F \sim 8.0)$, $\mathrm{CP} / \mathrm{IP}(F \sim 4.4)$ and $2-\mathrm{MP}(F \sim 4.2), \mathrm{F}$ is reasonably small indicating that all three liquids form fragile glasses if cooled appropriately.

\subsection{Reproducibilty of viscosity data}

The reliability of the viscosity data is due to the temperature stability $\Delta T$ of the cryostats used. The uncertainty $\delta \eta$ of $\eta$ is given by $\delta \eta \approx(\partial \eta / \partial T) \times \Delta T$. For the older cryostat $(\mathrm{A})$ the uncertainty of the temperature was $\Delta T \approx \pm 0.15 \mathrm{~K}$, which was mainly due to a temperature gradient within the cryostat. The corresponding errors $\delta \eta$ for the solvents measured in cryostat (A) are smaller than $\pm 3.7 \%$ for 2 -MP, $\pm 0.4 \%$ for $\mathrm{CP}$ and $\pm 3.6 \%$ for both $\mathrm{CP} / \mathrm{IP}$ mixtures at the lowest respective temperature measured (see Table 1). For the solvents 1-Prop, Isooct, and ct-DMCH, which were studied in the improved cryostat (B) with a significantly better spatial temperature stability, the total uncertainty of the temperature was $\Delta T \approx \pm 0.05 \mathrm{~K}$, which is mainly due to the uncertainty in the electronic temperature read-out. The resulting uncertainties for the respective solvents are smaller than $\pm 1.0 \%$ for 1 -Prop, $\pm 0.4 \%$ for Isooct, and $\pm 2.5 \%$ for all ct-DMCH mixtures at the lowest respective temperature measured. For the two-component liquids $\mathrm{CP} / \mathrm{IP}$ and ct-DMCH an additional error may result from the composition, which is discussed below.

\subsection{Reliability of density data}

The accuracy of the density measurements was limited by the following estimated uncertainties: the temperature dependence of the pycnometer volume $(\sim 0.02 \%)$, readings of the height of the liquid in the capillary $(\sim 0.02 \%)$, no correction for the shape of the meniscus $(\sim 0.02 \%)$, uncertainty of the vapour phase correction $(\sim 0.01 \%)$, uncertainty of the temperature $(\sim 0.05 \%)$. Moreover, the literature values $\varrho_{20}{ }^{\circ} \mathrm{C}$ for the pycnometer calibration refer to air-saturated liquids, whereas the present measurements were performed using degassed liquids, which introduces an additional uncertainty. In conclusion, the absolute accuracy of the density data is of the order of $\pm 0.15 \%$.

\subsection{The glass-forming binary mixtures ct-DMCH and CP/IP}

For the solvent mixtures ct-DMCH and CP/IP no temperature-dependent viscosity measurements could be found in the literature. It is known, however, that the 30:70 mixture $\mathrm{CP} / \mathrm{IP}$ stays viscous even at a temperature of $\sim 90 \mathrm{~K}$ [19], which was the reason for using this specific mixture in previous investigations 
on bimolecular photophysical processes [20]. The effect of mixture composition on the viscosity was studied for both liquids by deliberately altering the respective mole fractions. In the case of ct-DMCH the 50:50 composition of cis/trans was compared with ratios of 45:55 and 55:45 in the high viscosity region (see Fig. 1). For CP/IP the composition 30:70 of CP and IP was compared to that of 27:73 (Fig. 2). For all mixtures with varied mole fractions of the components $\eta(T)$ was equally well described by a single VTF-function as shown by the relative deviation $\Delta \eta$ in the lower parts of Figs. 1 and 2 (symbols "-" and "|" for ct-DMCH(2) and (3), and " $x$ " for CP/IP(2)). In the corresponding graphs the relative deviations $\Delta \eta=\left(\eta_{\text {mixture(1) }}-\eta_{\text {mixture }(k)}\right) / \eta_{\text {mixture }(1)}(k=2,3$ for ct-DMCH and $k=2$ for CP/IP) are shown using the symbol "•".

$C t-D M C H$ : A lower cis-1,4-DMCH content yielded larger viscosity values than the 50:50 mixture for all temperatures between 126 and $143 \mathrm{~K}$. The average increase for ct-DMCH(2) was found to be $3 \pm 1 \%$. For a lower trans$1,3-\mathrm{DMCH}$ content no significant deviation in $\eta$ from the 50:50 mixture was observed (see Fig. 1). Considering the uncertainty of the mole fraction of $\pm 0.5 \%$ in the preparation of the mixtures together with the total uncertainty of the viscosity measurement of $\pm 2.5 \%$ in the low temperature region, it is justified to assume the observed deviation of $\eta(\mathrm{ct}-\mathrm{DMCH}(2))$ from $\eta(\mathrm{ct}-\mathrm{DMCH}(1))$ to be still within the error limit of the measurement. This is also supported by the fact that the fragility parameter $F$, obtained from the VTF fit for ct$\mathrm{DMCH}(2)$ and ct-DMCH(3) only varies by $2.7 \%$ in accordance with the error limit, i.e. $E_{3} / R$ and $T_{0}$ deviate by less than $2.5 \%\left(E_{3} / R(2.4 \%), T_{0}(0.4 \%)\right)$.

$C P / I P$ : For the mixture with the lower $\mathrm{CP}$ content, $\mathrm{CP} / \mathrm{IP}(2)$, a systematic decrease of $\eta$ of up to $\sim 3 \%$ is observed in comparison to the CP/IP 30:70 mixture. This deviation is also well within the error limit of the measurement, considering an overall uncertainty of $\pm 3.6 \%$ in the viscosity and $\pm 0.5 \%$ in the preparation of the respective mole fractions. The systematic behaviour of the deviation could be based on the fact that $\eta(\mathrm{CP} / \mathrm{IP})$ was measured in the prototype cryostat $(\mathrm{A})$ with a more significant temperature gradient in the sample liquid.

In conclusion, a small change in the mixtures' compositions seems uncritical for the corresponding viscosity, if they are measured with the accuracy of the present investigation.

\subsection{Comparison with literature data on viscosity for the one-component liquids}

The available viscosity data for 1-Prop, Isooct, 2-MP and CP from the literature are compared with the results presented here and are briefly discussed.

1-Propanol: Literature data are available for the liquid 1-Prop over a wide temperature range between $\sim 369 \mathrm{~K}$ and $\sim 103 \mathrm{~K}[21-24]$ well into the supercooled region. The data span a viscosity range of approximately 12 orders of magnitude. The values by Thorpe and Rodger [21], who measured be- 
tween $\sim 213$ and $369 \mathrm{~K}$ follow a simple Arrhenius behaviour covering the low viscosity region between $\sim 1.7$ and $32 \mathrm{mPa}$ s (see also [25]). Tammann and Hesse [22] performed viscosity measurements in the non-overlapping range from $\eta(177 \mathrm{~K}) \approx 233$ to $\eta(138 \mathrm{~K}) \approx 1.59 \times 10^{5} \mathrm{mPa}$ s using a fallingball-viscosimeter. Denney [23] employed a rotating cylinder method to extend the viscosity region further to $\eta(121 \mathrm{~K}) \approx 1 \times 10^{7} \mathrm{mPa} \mathrm{s}$, before Ling and Willard [24] studied the high viscosity range from $\eta(131 \mathrm{~K}) \approx 1.9 \times 10^{6}$ up to $\eta(104 \mathrm{~K}) \approx 1.5 \times 10^{12} \mathrm{mPas}$ in the glass transition region by penetration viscosimetry.

The viscosities presented here close the gap between the viscosity measurements in [21] and in [22,23]. The agreement between the present data and those in [21] is better or similar to $1 \%$. In the region from 138 to $177 \mathrm{~K}$, however, the viscosity data in [22] are systematically too small, deviations range from $8 \%-30 \%$. This discrepancy can be understood on basis of the large temperature variation of up to 3.5 degrees Kelvin reported in [22]. The low viscosity regime in [23] $(149 \ldots 152 \mathrm{~K})$ still overlaps with the present data and agree within the accuracy of $20 \%$, stated in [23]. In Fig. 2 of [23] an extrapolation of viscosity data into the high temperature regime is shown, which also supports the finding that the values measured by Tammann and Hesse are signifcantly too small. From the measurements by Ling and Willard [24] it can be seen that, at temperatures below $150 \mathrm{~K}$ our description of $\eta(T)$ by two Arrhenius terms (see Fig. 2 and Table 1) no longer holds and a VTF term is required for an adequate description. Thus only three extrapolated values are given in Tables 2 for 1-Prop. Ling and Willard measured $\eta_{1 \text {-Prop }}$ to a minimum temperature of $103.5 \mathrm{~K}$ (assuming a constant density of $0.91 \mathrm{~g} \mathrm{~cm}^{-3}$ in their evaluation procedure) for which a viscosity of $1.5 \times 10^{12} \mathrm{mPa}$ is reported. A discussion of the dynamics of 1-Prop in the glass transition region in conjunction with dielectric relaxation measurements can be found in Refs. [26-28].

2-Methylpentane: In [29] viscosity data on the glass-forming liquid 2-MP are reported between $\eta(96.5 \mathrm{~K}) \approx 5.2 \times 10^{6}$ and $\eta(80.5 \mathrm{~K}) \approx 6.3 \times 10^{13} \mathrm{mPa} \mathrm{s}$ in a temperature region well below the one presented here. Greenspan and Fischer [30] report relative viscosities obtained with penetrometric measurements in a similar temperature range $(\sim 94 \ldots 101 \mathrm{~K})$.

Cyclopentane: The viscosity of $\mathrm{CP}$ can be compared to results by Fischer and Weiss [31], who measured the dynamic viscosity of CP between 250 and $315 \mathrm{~K}$. The viscosities in [31] are systematically larger, the densities systematically smaller than our values, the deviation however is always $<1 \%$ and $<0.1 \%$ respectively. Assael and Dalaouti [32] also studied the pressure dependence of the viscosity of CP between 210 and $310 \mathrm{~K}$. Their data also agree with our results to better than $1 \%$, which corresponds to the error limit stated in [32] for that temperature region.

Isooctane: The pressure dependence of the density and viscosity of Isooct was studied by Padua et al. [33, 34] for temperatures between 198 and $348 \mathrm{~K}$. The densities and viscosities measured at normal pressure agree with our data 
in the overlapping temperature region within $<0.2 \%$ and $<2 \%$, respectively, except for the lowest temperature reported in [33], where the deviation is $\approx+0.4 \%$ and $\approx+8 \%$. The temperature uncertainty in [33] is stated to be somewhat smaller than in the present investigation. A discrepancy of $<2 \%$ in the viscosity can be explained by the difference in temperature stability in combination with the reported uncertainty in the pressure at normal conditions. The differences of values $\eta(198 \mathrm{~K})$ is however not understandable on basis of the data reported, since even an error as large as $\pm 0.15 \mathrm{~K}$ in the temperature stability would only cause a discrepancy of $<1 \%$ at $198 \mathrm{~K}$.

\section{Summary}

The dynamic viscosity $\eta(T)$ of the 50:50 mixture of cis-1,4/trans-1,3dimethylcyclohexane was measured by capillary viscosimetry over a range of almost seven orders of magnitude. $\eta(T)$ can be well described by $f(T)$ the sum of two Arrhenius terms and one Vogel-Tammann-Fulcher term, the latter dominates at high viscosities. This description also holds to describe the temperature dependence of $\eta$ of several other frequently used organic solvents (1-propanol, 2-methylpentane, cyclopentane, isooctane and the mixture cyclopentane/isopentane), whose dynamic viscosity was measured over $\sim 1-5$ orders of magnitude. The relative accuracy of the measured viscosity data ranges from $\pm 0.4 \%$ at high temperatures to $\pm 3.7 \%$ at low temperatures. Systematic deviations of $f(T)$ from $\eta(T)$ are typically $<2 \%$. Changes in the mole fraction of the binary mixture of $<5 \%$ result in a deviation of the viscosity within experimental error. The temperature dependence $\varrho(T)$ of the density of the solvents is also reported.

\section{Acknowledgement}

We would like to thank Dr. U. Nickel for her comments on the manuscript, Dr. P. Borowicz (MPI für Biophysikalische Chemie, Göttingen) for his assistance and Prof. J. Troe (Institut für Physikalische Chemie, Universität Göttingen) for strongly supporting this work.

\section{References}

1. B. Nickel, P. Borowicz, A. A. Ruth, and J. Troe, Phys. Chem. Chem. Phys. (2003) to be published.

2. B. Nickel, H. E. Wilhelm, and C. P. Jänsch, Optics \& Spectr. 83 (1997) 541.

3. J. A. Riddick, W. B. Bunger, and T. K. Sakano, Organic Solvents, $4^{\text {th }}$ edition (Vol. II of "Techniques of Chemistry", Ed. A. Weissberger), Wiley-Interscience, New York (1986) and references therein.

4. L. Ubbelohde, Zur Viskosimetrie, $7^{\text {th }}$ edition revised by G. H. Göttner and W. Weber, S. Hirzel, Stuttgart, 1965. 
5. J. F. Swindells, R. Ullman, and H. Mark, Determination of Viscosity, in Physical Methods of Organic Chemistry, Vol. I, Part I., Ed. A. Weissberger, Interscience Publ. New York (1965), Chapt. XII, p. 689 ff.

6. Landolt-Börnstein, New Series, Editor in Chief: O. Madelung, Units and Fundamental Constants in Physics and Chemistry, Subvolume a: Units in Physics and Chemistry; H. Bauer, 2.3.1.12 Viscosity.

7. H. Lesche, D. Klemp, and B. Nickel, Z. Phys. Chem. 141 (1984) 239.

8. A. A. Ruth, B. Nickel, and H. Lesche, Z. Phys. Chem. 175 (1992) 91.

9. G. Harrison, The dynamic properties of supercooled liquids, Academic Press, London (1976).

10. P. Taborek, R. N. Kleiman, and D. J. Bishop, Phys. Rev. B 34 (1986) 1835.

11. S. S. N. Murthy, J. Chem. Soc. Faraday Trans. 285 (1989) 581.

12. A. J. Barlow, J. Lamb, and A. J. Matheson, Proc. Roy. Soc. A 292 (1966) 322.

13. Beilstein Handbuch der Organischen Chemie, Vierte Auflage, Drittes Ergänzungswerk, Band 5, Teil 1, H.-G. Boit, Springer-Verlag, Göttingen (1963), p. 100, 103.

14. National Institute of Standards and Technology, Standard Reference and Materials Program, SRM 2214 (Isooctane), Gaithersburg, Maryland, Feb. 2001.

15. S. S. N. Murthy, Thermochim. Acta 359 (2000) 143.

16. M. Tyagi and S. S. N. Murthy, J. Chem. Phys. 114 (2001) 3640.

17. C. A. Angell, J. Non-Cryst. Solids 131 (1991) 13.

18. C. A. Angell, Science 267 (1995) 1924.

19. J. Timmermans and J. Naveau, Bull. Soc. Chim. Belg. 67 (1958) 560.

20. D. Klemp and B. Nickel, Chem. Phys. Lett. 97 (1983) 66.

21. T. E. Thorpe and J. W. Rodgers, Phil. Trans. Roy. Soc. London 185A (1894) 397.

22. G. Tammann and W. Hesse, Z. Anorg. Allg. Chem. 156 (1926) 245.

23. D. J. Denney, J. Chem. Phys. 30 (1959) 159.

24. A. C. Ling and J. E. Willard, J. Phys. Chem. 72 (1968) 3349.

25. E. N. C. Andrade, Philos. Mag. 17 (1934) 698.

26. F. Stickel, E. W. Fischer, and R. Richert, J. Chem. Phys. 104 (1996) 2043.

27. C. Hansen, F. Stickel, T. Berger, R. Richert, and E. W. Fischer, J. Chem. Phys. 107 (1997) 1086.

28. B. Schiener and R. Böhmer, J. Non-Cryst. Solids 182 (1995) 180.

29. A. C. Ling and J. E. Willard, J. Phys. Chem. 72 (1968) 1918.

30. H. Greenspan and E. Fischer, J. Phys. Chem. 69 (1965) 2466.

31. J. Fischer and A. Weiss, Ber. Bunsenges. Phys. Chem. 90 (1986) 896.

32. M. J. Assael and N. K. Dalaouti, High Temp. High Press. 32 (2000) 179.

33. A. A. H. Pádua, J. M. N. A. Farcleira, J. C. G. Calado, and W. A. Wakcham, Int. J. Thermophys. 15 (1994) 229.

34. A. A. H. Pádua, J. M. N. A. Farcleira, J. C. G. Calado, and W. A. Wakcham, J. Chem. Eng. Data 41 (1996) 1488 (see also references therein). 